\title{
Developing a Collaborative Learning Support System for a Natural Protected Area
}

\author{
Adele Celino and Grazia Concilio \\ Department of Architecture and Town Planning, Polytechnic of Bari, via Orabona 4, \\ 72019 Bari, Italy \\ \{a.celino,g.concilio\}@poliba.it
}

\begin{abstract}
The proposed contribution presents the results of an on going research work aiming at the implementation of a knowledge based system devoted to supporting the local government and the citizenship during the setting up process of a Natural Protected Area in Southern Italy. The system architecture integrates Group Decision Support Technology already available on the enterprise software market with tools enabling a dynamic representation of organizational memory. Organizational memory, structured within information systems supporting decision-making and action in organizational environments, can be a useful mean for developing multilevel (individual, of group, organizational) collaborative learning. Starting from the case study -the process for setting up the Gravina Natural Protected Area-, the paper describes the system's architecture and discusses some problematic issues related to: expert and nonexpert knowledge acquisition and representation; possible dynamic representation of organizational memory, creation, use and storage of decision/learning histories, and dimension and relevance of memory.
\end{abstract}

\section{Introduction}

The present contribution refers to an on going research work funded by the Italian National Research Council in order to develop GraviCS, an argumentative information system oriented towards cognitive management of the decision making process [6] and developed to support decision making and future scenario building in the setting up process for a Regional Natural Park. Such a process appears very interesting in terms of learning opportunity since it induces relevant modifications in the relation between the environment and its settled communities.

When managing decision making processes in environmental planning, that is, when dealing with the relevant role of knowledge and the related knowledge actors, problematic issues are related to: the models and methodologies for building decision options, the exploitation of involved interests and values, and the exploration of elements which are fundamental for decision making. These themes represent focus points when designing and implementing DSSs' architectures. With regards to problem-oriented DSSs, most applications in environmental planning refer to environmental resource monitoring and management. Process-oriented DSSs, when developed in environmental planning domains, aim at supporting interaction between decision makers and facilitating the management of conflicts which often emerge within 
the involved decision making structures. These latter are, moreover, fluid, interpretative, scarcely limited by formal organizational boundaries and are destined to selfdefine their structure during the evolution of the action process.

In designing the GraviCS system, we try to integrate the two approaches described both designing and implementing a DSS whose main purpose is to organize a dynamic representation of the organizational memory considered means not only of accumulating and exchanging knowledge but also to explore the operability of transient-scenario. A transient-scenario represents one of the temporary images of the process-scenario that is a scenario (in our case an environmental one) which evolves together with its related decision making system and with the community action itself.

Starting from a description of the organizational context of the Gravina (the naturalistic resource being the emergence of the protected area), the paper: i) discusses some problematic issues related to the possible dynamic representation of organizational memory, to the creation, use and storage of decision/learning histories, and to the dimension and relevance of memory; ii) introduces the concept of processscenarios; iii) describes the decision support system's architecture developed in order to support learning mechanisms in a complex organizational environment in which local government and the citizenship work together.

\section{From the Operational Context to the System}

The research work started after the publication of the "preliminary studies" for the Natural Protected Area of the Gravina: "preliminary studies", as they are called in the Apulia Regional Law (n.19, 1997), represent a preliminary in-depth analysis of the territory devoted to the implementation of a Natural Protected Area. The Regional Law also defines the formal procedure for the park setting up: after the publication of the "preliminary studies", the regional law imposes the organization of the so called "preconferenze", a sort of public meetings which are not only devoted to a consultancy process but also are searching for political agreement among stakeholders. During such "preconferenze", a preliminary agreement on the park boundaries is ratified but cautionary norms (to be respected until the plan is approved) are not discussed. These are based on a standard grid, very little scarcely context-oriented and highly disruptive in terms of effects on the community due to the fact they cannot have short-term effects. During "preconferenze" period, a great role is given to negotiation processes but no space is left to mutual learning: in this phase even information exchange is inhibited.

While preparing "preconferenze", relevant roles are played by soft forms of local intermediation (based on intervention models characterized by high informality), influence by media, on-going planning processes, politicians' interventions. Elements affecting the parks setting up processes are: the strong political will (sometime the momentary composition of different groups with different behavioural logics), the administrative fragmentation, strong or spread interests.

The roles the local government can play in the process may be different with regards to different action strategies and political goals: 1) the regional authority may start the park setting up process leaving the local municipality and community consultancy no opportunity for consolidating their own knowledge or their own opinion on the park issues (this procedure is coherent with the current normative framework); 2) the local 
government and the community interact, discussing the way the Gravina territory can be environmentally protected, and eventually propose to the regional authority to start the park setting up process; 3) the local government and the community interact, discussing the way the Gravina territory can be environmentally protected, and decide to wait for the regional authority to start the park setting up process.

In the end, the local government can play the role of park setting up proponent or be a passive actor of the process.

After long interactions with the Regional Natural Park Office and with the local government, in agreement with the latter, an interactive process coherent with perspective 2) was started. Supported by the local government 24 actors were selected who are concerned with the environmental and territorial themes chosen. Each of the actors was interviewed singularly in order to build individual text profiles describing the role he/she may have in the interaction in terms of cognitive contribution, experiences, and potential links with a wider portion of the community. At present we have planned the first two preliminary vis-à-vis meetings: the first will be oriented to a presentation of the initiative and participants; during the second meeting, the interaction support system GraviCS will be presented and a work agenda will be developed collaboratively.

\section{Planning the Initiative}

\subsection{The Organizational Field and Its Loose Coupling}

The GraviCS research work is carried out within the political and procedural framework described above and tries to give a formal value to the interactive phase preceding the "preconferenze" with a triple goal: i) to enrich the cognitive dimension of this phase; ii) to enable a rapid process of plan design in case of a positive outcome of the interactive process, thus reducing the duration of the cautionary norms; iii) to enhance an active role of the local community (citizens but also local government) rather than a reactive one.

Because the organizational field of the Gravina, as many others complex field, does not coincide with the existing strategic and conflict arena [10] but it includes, far as possible, actors who can potentially play a relevant role in the collective management of the natural protected area, a preliminary analysis was needed in order to define its boundaries and the structure.

In order to define the potential boundaries of the organizational field, it was useful to recognize some of its principal actors, they being also potential users of GraviCS; the structure of the organizational field suggested interesting elements for some GraviCS tasks.

In particular, individual interviews were useful in order to gain information on the field structure defined as interaction degree and nature of the inter-organizational structure [12]. The interviews, in effect, not only supplied the profiles of the field actors and described their potential roles (in terms of cognitive and experiential contribution inside and outside the field) in the interaction process, but also gathered useful information on institutions, sets of practices and other relational contexts. 
Looking at the interviews, the organizational field of the Gravina territory shows the existence of a loose coupling among actors and among development policies adopted over the last decades. The loose coupling is a characteristic of all the organizations and, in fact, of all open systems [14], [24], [19]; in the case of the organizational field related to management of the Gravina territory, this loose coupling assumes a special meaning.

According to a thesis proposed and examined by Di Maggio, in effect, the greater the concentration of resources on which organizations are based, the more structured their organizational fields are. In the case of the Gravina Natural Protected Area, the great resources concentration characterizing the field shows a strong potential structuring which is now a non-collaborative structuring. The coupling characterizing the examined organizational field are evidently ambiguous and show the overlapping of environmental planning and management interventions which are fragmented and uncoordinated.

Referring to some of Weick's observations on loosely coupled systems [24] we can recognize in the existing organizational field:

- several situations in which many and different means are used to produce the same output (ex. improvement of the environmental quality is the goal of programs for the Gravina world life protection, for fauna and flora conservation, for historical area rehabilitation, but also of initiatives and policies, oriented to the economic and tourist development, which aim at making the area attractive for potential users);

- the lack of coordination, which is mainly evident in the overlapping of plans, programs and policies sharing the same goals and aiming at the management of the same resources (the archaeological area, the urban area, the Gravina natural area and the areas for the famous wine production are all in the same geographical location but submitted to different norms and constraints);

- the lack of regional norms (the only regional plan, regulating spatial planning in the Puglia region, does not recognize the Gravina area as a relevant environmental location);

- the presence of some action networks which are highly connected but hardly interested in feedback.

The observed organizational field is characterized by a multilevel decision environment where the stakeholders interact and work together, though evidently independent of each other. In this way: i) there is a huge consumption of time and energy because of the disagreements on use of resources; ii) each single stakeholder selects and improves personal practices which aim at shared general objectives but produce overlapping outputs; iii) each single stakeholder organizes communication practices independent of the others; iv) there is an evident decentralization of practical norms.

The process supporting the setting up of the Natural Protected Area has to deal with an organizational field whose loose coupling limit and inhibit both the reactions to external inputs [25] and the diffusion/transmission of information and knowledge, and thus eventual collaborative learning processes. "By definition, a loosely coupled system is... harder to administer" [26].

Although the conditions described above may seem negative, they are exactly the conditions which enable an organization field: to resist sudden environmental changes; to develop a deeper sensitivity to the external environment; to adapt to local 
conditions; to tolerate "breaking points" of parts of the system without damaging the whole organization.

In the end, all the issues related to organizational fields which are loose coupled systems represent a set of relevant questions on the theme of transferring and generating knowledge within organizations. These questions become more and more relevant when the organizations are supported by information and technology tools which enhance the generative dimension (both structuring and un-structuring) of the field languages.

In collaboration with the Gravina local government and abandoning the idea to intervene into the park setting up process with a pre-defined organizational model, our research work aimed at supporting the organizational field to start an evolving process which depends on the history of its internal relations and is characterized by collaborative learning. In order to support such a process, we are also implementing a knowledge-based system that integrates information tools supporting group decision making with tools oriented to the dynamic representation of the organizational memory.

\subsection{The Organizational Memory}

Generally speaking, organizational memory means the set of information stored by an organization during its activities and necessary to make better informed decisions [23] This information is obtained as result of decisions already implemented and/or explored and are enriched by both individual knowledge and collective interpretations taken from argumentative dialogues.

When structured within information systems supporting the coordination of decisions and actions in organizational environments, organization memory can reveal to be a useful means to develop multilevel collaborative learning (individual, group, organizational), and therefore to support cooperation in multi-task and multi-users environments. Organizational memory, in effect, can facilitate the integration of organization actors, reduce transaction costs and adapt itself to external/internal changes [23]. Organizational learning is easier when individuals and groups, as the agents for knowledge transfer, somehow share cognitive systems and memories.

In information systems, organizational memory is based on systematic monitoring and storing of information streams; therefore, it represents one of the basic tasks of the system supporting the structural analysis of information exchange processes. Since organizations are able to extract meta-information from stored information, organizational memory can help to select actions towards learning but also identify reasons and mechanisms inhibiting it. Finally we can try to improve the learning process: by removing mechanisms recognized as negative and also by recalling those actions considered effective and productive.

In order to improve learning, organizational memory needs to be supported by an effective system. Weick suggests that if an organization has to learn something, the distribution and the accuracy of its memory become crucial characteristics [25]. In other words, an ideal organizational memory system has to be consistent and retentive, and to reach a good distributive capacity. Moreover it should not be an obstacle to creative and innovative activities.

Since the organizational field operating on the Gravina, is a loose coupled field, it presents an organizational memory similar to that of oral cultures: it is an easy modifiable memory, organized by spread knowledge and information sources (sometimes 
they are also independent), is scarcely retentive, and is able to store only what it needs for its balance -such balance often coincides with the organizational status-quo.

These characteristics are also characteristics which make the organization creative since they allow the actors of the organizational field to reinterpret the past in a retrospective way (the existence of loose coupling, in effect, can facilitate the combination and the recombination of knowledge-based resources in a way which is flexible and creative).

The "store" metaphor, as representation of memory is no longer adequate to such an organizational field: it needs to be supported in a way which is able to evolve together with the on-going process.

Information technology, for such cases, presents some advantages: it makes the memory contents explicit, modifiable and sharable when needed. By the use of information technology, information propagation within organizational fields with loose coupling can become possible.

\subsection{Dynamic Representation of Knowledge: The Use of Transient Constructs in Organizations}

In GraviCS the role of the organizational memory is to enable the exploration of the operability of transient-scenario (elements of such scenarios are: the park boundaries proposal, the proposal for implementation norms, the action agenda) in a space which is constrained by the existing organizational field and also by other forces governing spatial transformation but external to the field itself.

It is a space constrained by: norms and rules, institutional organizational structures, relationships among stakeholders (institutional and not), rooted practices which are now routines, and practices foreseen as desirable within the preliminary studies to protect natural and anthropic environments.

Organizational memory, therefore, is considered a combination of initial available knowledge of the organizational field (knowledge stored in documents, in practices, in procedures) and knowledge structured in the system itself and being able to describe how initial knowledge has been used by the users during the interactive process.

The system memory, therefore, should take into account both the initial knowledge (the base knowledge) and the process knowledge (which considers evolutions and changes occurring in procedural schemes of knowledge use. The process knowledge and that developed during interaction are evolving and need to be captured, formalized and diffused throughout the whole organizational field.

Concerning the above considerations, some of the cycles carried out in "virtual spaces" enable the users to be involved in a simulation experience; derived learning cycles are typically experiential since the actors are given the opportunities to reflect on the experience, to apply theories and concepts to their observations, and to identify new possible objectives and alternatives referring to the new knowledge continuously generated during interaction. Such mechanisms enable the transaction from one "transient-scenario" (and its supporting organizational structures) to the next one.

The series of organizational structures and transient-scenarios represent the set of transient constructs which are knowledge "containers" [17] facilitating experimentations and changes and whose tasks in the system are: 
- to represent knowledge dynamically through its changing (or disappearing) with time;

- to take a picture of the "current" knowledge (in the form of transient-scenario park boundary proposal, norms proposal, implementation agenda).

\section{GraviCS Architecture}

GraviCS aims at supporting collaborative learning to develop process-scenario for environmental planning in an argumentative, interactive environment. The system's architecture has been designed in order to integrate, in a GIS environment, IT tools traditionally oriented to group decision support and currently largely used in the market of enterprise software [8], [2] with instruments oriented to the representation of organizational memory described in the preceding paragraph.

The GIS environment, although considered a starting reference for the system implementation, represents neither its structural nor its functional heart. Actually, the GIS environment represents a sort of landscape where the system is developed and, with regards to its fundamental functions, it is no more than a tool supporting: i) the visualization of cartographic information, ii) the geo-referring and exploration of hypertexts; iii) the geo-exploration of the data-base.

The GraviCS architecture has been designed in order to enable its users and the system manager to configure the system coherently with support needs, which are evolving together with the process [3], [7], [16]. The system, in fact, initially makes available only some basic functions; it is the user/s to explore, and eventually activate, new tools/functions that are always available but never imposed automatically by the system. This perspective has been considered relevant coherently with the need to develop a support system in a bottom-up approach [4] that is through a close collaboration with the organizational context that uses the system. This perspective has not been translated, within this work through a system converging toward a definitive architecture, but more referred to the idea of developing a system that can evolve together with the evolving interaction/decision process.

GraviCS is a web-based system and is substantially divided into two main modules (figure 1). The first module, with limited access (by the use of passwords) represents a sort of intranet where assistance is supplied to the interaction of a small group; in this module the eventual access of expert consultants is also managed (users can ask for the intervention of further participants whose cognitive contribution is consensually considered necessary for discussion on specific problems).

The second module represents the web interfaces of the system and currently its design still has to be started: we shall consider making such interface active only when, during the park setting up process, the interaction requires enlargement to a wider community whose interaction goals and roles are unknown (not necessarily declared, as it is the case with the small group accessing the system with a password).

\subsection{Operational Tasks of the System}

The system architecture has been designed considering the relevant goal to support organizational learning. With regards to this perspective the main challenges in de- 
signing GraviCS were represented by the system's functions oriented to the creation and the management of the organizational memory due to the need to keep in the system and to make available to users information and knowledge considered relevant for the evolution of the decision making process.

Therefore, the design and implementation work, related to the first module, aimed at two main operational goals:

- transferability of information and knowledge

- accessibility to the interaction process
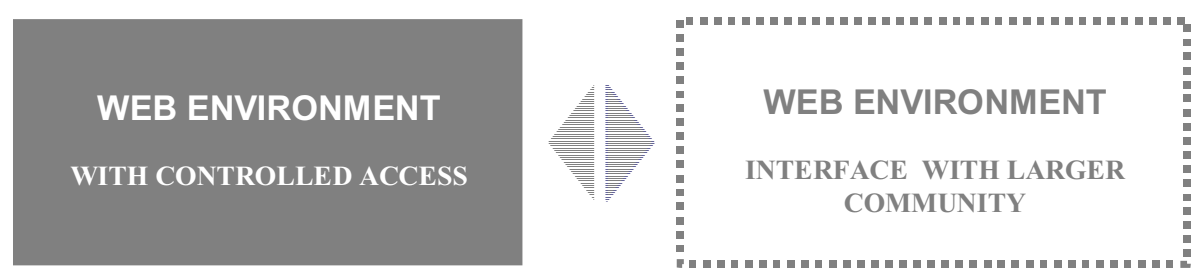

Fig. 1. Two macro-modules for GraviCS

The first goal not only poses the relevant problem of archiviation of information and knowledge but mainly that of information and knowledge retrieving from the archive although this archive is rapidly growing in dimension and diversification [9]. The second goal, instead, poses the problem of a dynamic representation of the organizational memory, that is a problem of immediate or slow understanding of the advancement level of the interactive process with regards to its origin, its ultimate goals, the interaction contents, and the different cognitive position of the participants. With regards to the first goal, Conklin [9] evidenced that traditional tools for information/knowledge acquisition and archiviation (like those available in enterprise management softwares: e.mail, Lotus Notes, ...) fail in the creation of an organizational memory because of inadequacy in indexing, that is inadequacy to organize the memory itself. In facing this problem, the system privileges an organization based on two main kinds of relations: semantic relations and geographic relations. Semantic relations are represented in the system through hyper textual links, geographic relations are introduced through a geographic indexing linking information and knowledge to maps. In some cases these different relations can contemporarily characterize a relation: two approaches in the archive navigation are possible and are always available to the user, but also parallel navigations in documents and maps are available [1].

The second goal takes into consideration the need to facilitate users' access into the interactive process, both when they are "ordinary" or "extraordinary" participants: the more rarely the user uses the interaction space, the more accessibility is reduced. For this second goal the traceability and the transparency of the process becomes relevant [18]. Process traceability is relevant in order to enable process access to extraordinary users, that is to newcomers (Märker and Pipek's perspective), and in our case it is a basic issue, with regards to the system goal to support learning mechanisms, in order to facilitate creation of feedback and reflection. In this sense two different synthetic representations of the process trace have been introduced in form of causal maps. 
The first representation, of more general nature, is a sequential diagram of the formal process for park setting up: the system of sequential events, constraints, and actors of the setting up process. This first map ( $I^{\text {st }}$ level map), a non-dynamic map, aims at making role and location of the current interactive phase explicit within the whole process as it is structured by the law: this is extremely useful at the start of the interactive process since it is not explicitly included into the formal process.

The second representation ( $2^{\text {nd }}$ level map), unlike the first, has a dynamic framework, that is it changes together with the interaction process: $2^{\text {nd }}$ level maps visualize the sequences of causal argumentations [20] that explain the transaction within the process from one process condition to the next one which is more.

\subsection{Tasks and Functions of the Implemented System}

GraviCS architecture concerning the first macro-module, is shown in fig.2. It is substantially based on two modules: an archive, containing the normative data base, all the documents of the preliminary studies (expert studies), and the process memory; and a work space, the module supporting the argumentative dialogue[15].

A further module is a sort of container of useful instruments (tools box): it is accessible both by users and system managers and contains instruments which are eventually useful for interaction but are made available only if their use is expressly required by users or suggested by the system manager.

The implemented system supports the transition from one state of the interaction process to the next: the transition from one state to the other is represented by a transaction (positive, negative or partial) of a causal argumentation and is formalized by the system through the transition from one transient-scenario to the other. A sequence of transient-scenarios is considered to give shape to the process-scenario. Causal argumentations are produced and discussed in an asynchronic interaction environment, or in a synchronic environment (with forum and chat opportunities), or also in vis-à-vis interaction spaces.

The Archive represents the space where three functions are implemented: exploration, archiviation, and visualization. All the three functions are supported by the hypertextual organization of the documents and by geographical indexing.

The process memory contains $1^{\text {st }}$ and $2^{\text {nd }}$ level causal maps. In particular, the $2^{\text {nd }}$ level maps are connected to the documentation of each transient-scenario to which they refer through the hypertexts and the geographical indexing. The process memory is updated every time a transaction of a causal argumentation is completed.

Very often, argumentative processes become dialogues between stakeholders [22]. The workspace aims at supporting these dialogues on the current transient-scenario. Argumentative dialogues represent a possible space where informal knowledge can be made explicit. Informal knowledge acquisition is somewhat problematic since the dialogues are informal themselves and are not recorded. The main task of this module is, therefore, to record structured dialogues through the creation of a framework of observations/argumentations and related comments. The former are producible in the individual environment (asynchronic space) and can have geographic indexing (if they are derived from a reflection on the park boundaries) or be mutually related through hypertext links (if they refer to norms or to the implementation agenda). To each observation/argumentation, users can attach a set of comments: observation/argumentation and related set of comments represent the starting point of fo- 
rum/chat discussions; forum, that can be remote or vis-à-vis, can be accessed also by extraordinary users (invited experts, consultants, institutional representatives, ...), eventually required by the group. To the active transient-scenario, in the $i^{\text {th }}$ current state, a set of causal argumentation (based on observations/argumentations and related comments) is associated; within the forum/chat environment the causal argumentation can activate the transition from the $i^{\text {th }}$ state to the state $i+1$ when the transaction is completed.

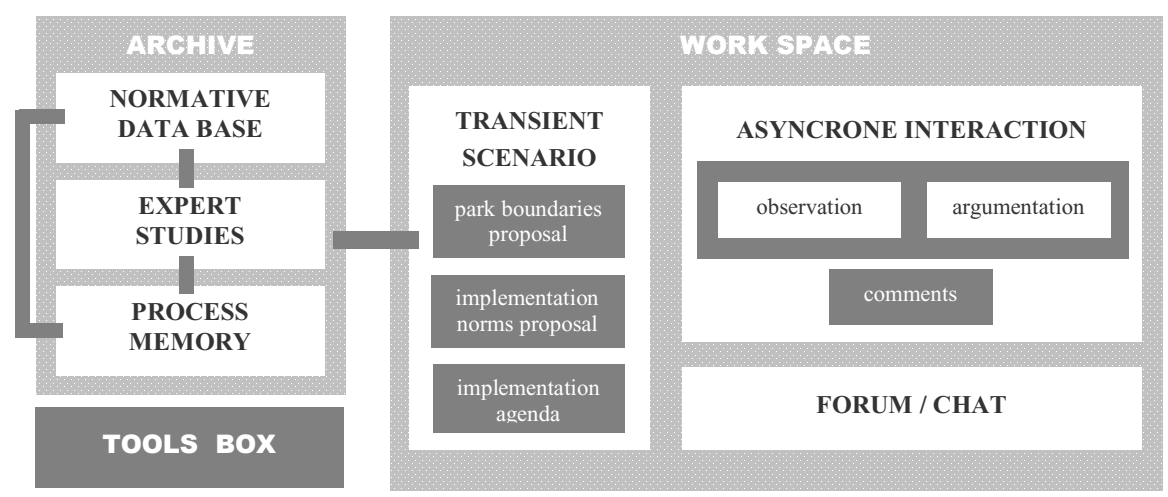

Fig. 2. GraviCS architecture

The Tools Box represents the space where many instruments supporting the interaction work and the system management are located. Basically, the box contains three categories of tools: interaction tools, representation tools and evaluation tools. Interaction tools are software able to create forum/chat environments, to supply remote support for mailing and interviewing. Representation is supported by software working in GIS environment and support map editing; representation tools are also instruments supporting causal maps production when they are developed both by users or automatically through text-analysis [5], [13]. The latter option is especially useful for the automatic up dating of $2^{\text {nd }}$ level maps, starting from textual recording of argumentative dialogues. Finally, evaluation tools are software supporting multicriteria decision making in group environment (Expert Choice e Naiade).

\section{Future Perspectives}

GraviCS has been developed in order to support both mediated and vis-à-vis interaction and to leave to the users the opportunity to ask and plan for different interactive conditions. Moreover, this flexibility appears relevant also with regards to learning opportunities due to the need to record the processes of information and knowledge creation, exchange and selection in whatever interaction conditions they are needed to be carried out. 
As already announced in the introduction, the research presented in this paper is still on going. We are now completing the implementation of GraviCS. In implementing GraviCS, we are trying to leave the implemented architecture as flexible as possible in order to have the possibility to fit the system to the planned agenda (the agenda will be discussed during the next meeting with the small group of stakeholders).

Many problems still feed wide areas of reflection and suggest possible directions for further research. The most relevant appears the problem of dynamically representing the organizational memory through both causal maps and transactions of causal argumentations. This approach needs to be tested with regards to the opportunity to evaluate the activation of learning mechanisms and their eventual progress.

The greatest challenge in this sense can be considered the dynamic approach in organizational memory management and in the representation of the decision making process. In order the system to be effective in this sense, it is necessary that system and users develop the same approach in building dynamic views of the process and of its evolution in the system representation: this asks for a deep flexibility of the system which, although many efforts, still does not really appear satisfactory in this sense.

Future changes in the system architecture and functions need to be planned coherently with the feedbacks deriving from the tests of the system in the users community and with the first attempts that will be done in order to recognize whether it is useful or not to support in some way learning process not limited to the individual level.

\section{References}

1. Barbanente, A., Cuscito, P., Maiellaro, N.: Expert system and hypertext for development control. Proceedings of 13th European Conference on Education in Computer Aided Architectural Design in Europe, Palermo (1995)

2. BLENKS: In-Team. http://www.blenks.com/inteam/ (last access Feb. 2002)

3. Bolloju, N., Khalifa, M., Turban, E.: Integrating knowledge management into enterprise environments for the next generation decision support. Decision Support Systems 33 (2002) 163- 176

4. Borri, D., Concilio, G.: Sistemi intelligenti multiagente di supporto alle decisioni. Atti della XX Conferenza Italiana di Scienze Regionali (AISRe), Piacenza (1999)

5. Carter, L.: Argument in hypertext: Writing strategies and the problem of order in a nonsequential world. Computers and Composition 20 (2003) 3-22

6. Chen, J.Q., Lee, S.M.: An exploratory cognitive Dss for strategic decision making. Decision Support Systems, 36 (2003) 147-160

7. Chuang, T., Yadav, S.B.: The development of an adaptive decision support system. Decision Support Systems 24 (1998) 73-87

8. Concilio, G., Kersten, G.: Information Technologies for Environmental Decision-Making in Networked Societies. Plurimondi (2003) forthcoming

9. Conklin, J. Designing Organizational Memory: Preserving Intelligent Assets in a Knowledge Economy (2001). http://cognexus.org/dom.pdf (last access october 2003)

10. DiMaggio, P.J.: Constructing an organizational field as a professional project: The case of U.S. art museums. In: Powell, DiMaggio (eds.): The New Institutionalism in Organizational Analysis. University of Chicago Press, Chicago (1991) 267-292

11. DiMaggio, P.J.: Culture and cognition. Annual Review of Sociology 23 (1997) 263-287 
12. DiMaggio, P.J., Powell, W.W.: The iron cage revisited: Institutional isomorphism and collective rationality in organizational fields. In: Powell, DiMaggio (eds.): The new institutionalism in organizational analysis. The University of Chicago Press, Chicago (1991) 63-82

13. Elhadad, M.: Using Argumentation in text generation. Journal of pragmatics 24 (1995) $189-220$

14. Glassman, R.: Persistence and Loose Coupling in Living Systems. Behavioral Science 18 (1973) 83-98

15. Karacapilidis, N., Papadias, D.: Computer supported argumentation and collaborative decision making: the HERMES system. Information Systems, 26 (2001) 259-277

16. Kersten, G., Mikolajuk, Z., Gar-on Yeh, A. (eds.): Decision Support Systems for Sustainable Development: A resource book of methods and Applications. Kluwer Academic Publishers, Boston (1999)

17. Lanzara, G.F.: Shifting stories. Learning from a reflective experiment in a design process. In: Schon, D.A. (ed.): The Reflective Turn. Teachers College Press, Columbia University, NY (1990) 285-320

18. Märker, O., Pipek, V.: Computer Supported Participation in Urban Planning from viewpoint of Communicative Planning Theory. Proceedings of IFIP 8.5 - Advances in Electronic Government, Zaragora Spain, (2000), 43-58

19. Orton, J. D., Weick, K.: Loosely Coupled Systems: a Reconceptualization. Academy of Management Review 15 (1990) 203-223

20. Östermeier, U., Hesse, F.W.: Verbal and visual causal arguments. Cognition 75 (2000)65104

21. Scott, R. W.: Institutions and Organizations. Sage, Thousand Oaks, CA. (1995)

22. Sigman, S., Liu, X.F.: A computational argumentation methodology for capturing and analysing design rationale arising from multiple perspectives. Information and Software Technology 45 (2003) 113-122

23. Walsh, J. P., Ungson, G.R.:, Organizational Memory, Academy of Management Review, $16 / 1$ (1991) 57-91

24. Weick, K.E.: Educational Organizations as Loosely Coupled Systems, Administrative Science Quarterly 21 (1976), 1-19

25. Weick, K.E.: The Social Psychology of Organizing. Addison-Wesley, Reading, MA (1979)

26. Weick, K.E.: Administering Education in Loosely Coupled Schools. Phi Beta Kappan, 63/10 (1982) 673-676 